\title{
FACTORS AFFECTING THE DEVELOPMENT OF COLLABORATIVE IMPROVEMENT WITH STRATEGIC SUPPLIERS
}

\author{
Rasmus Kaltoft ${ }^{1}$, Harry Boer ${ }^{1,2}$, Mariano Corso ${ }^{3}$, Frank Gertsen ${ }^{1}$, José Gieskes ${ }^{4}$, \\ Rick Middel ${ }^{4}$, Jacob Steendahl Nielsen ${ }^{1}$ \\ ${ }^{1}$ Center for Industrial Production, Aalborg University, Fibigerstræde 16, 9220 Aalborg, Denmark \\ ${ }^{2}$ Corresponding author. E-mail: hboer@iprod.auc.dk \\ ${ }^{3}$ Department of Management, Economics and Industrial Engineering, Politecnico di Milano, P.za L. da Vinci \\ 32, 20133 Milano, Italy \\ ${ }^{4}$ Faculty of Technology \& Management, University of Twente, P.O. Box 217, 7500 AE Enschede, The \\ Netherlands
}

\begin{abstract}
The research presented in this paper was aimed at increasing the current understanding of the process of developing collaborative improvement in Extended Manufacturing Enterprises (EME). Theory suggests a number of factors to affect that process, including shared sense of direction (i.e. vision), trust, power, and commitment. Based on action research of three EMEs involving a total of thirteen companies from five European countries, the present study identifies a number of additional factors. Factors exogenous to, but impacting, the collaboration are joint history and culture. Endogenous factors are approach to establishing the collaboration, project organisation, change and improvement competence, ways and modes of communicating, and political behaviour. Not only do these factors influence each other, they also strongly affect the development of collaborative improvement.
\end{abstract}

Keywords: Collaborative improvement, extended manufacturing enterprise, action learning, action research.

\section{INTRODUCTION}

The battlefield of competition is changing all the time, and companies are adapting themselves accordingly. While, until the 1980 s, vertically aligned operations prevailed [Hayes \& Wheelwright, 1984], the 1990s saw the emergence of horizontally aligned operations [Ghoshal \& Bartlett, 1995]. Today, companies increasingly link their internal processes with external suppliers and customers [Frohlich \& Westbrook, 2001] in so-called Extended Manufacturing Enterprises (EMEs). In effect, the battlefield of competition is increasingly moving from the level of individual firms to that of EMEs (Busby \& Fan 1993, Stock et al. 2000). Consequently, new approaches must be developed not only to enhance the business performance of EMEs, but also, in particular, the continuous improvement of their performance, relative to that of other EMEs (Michigan University).

Due to functional and, especially, geographical and time separations between partners involved, EMEs can hardly rely on organisational and managerial mechanisms supporting continuous improvement, while the Information and Communication Technology (ICT) needed to bridge these barriers is in its infancy. And then, even with suitable ICT-support, 
learning to improve collaboratively is a non-trivial, protracted process. Active collaboration between the firms involved is required in order to create and maximise synergy between the capabilities of the firms involved, while allowing each individual partner to realise its own strategic goals. This requires a well-developed capacity to learn, not only at the levels of individuals or companies, but also at the inter-organisational level. Until now there are neither clear theories nor tools to support inter-organisational learning in EMEs.

The CO-IMPROVE project addresses this need. Focusing on the learning required to enhance collaborative improvement of EME performance, the objective of the project is to develop:

- A Business Model, supported by:

- A web-based Software System, to enable and enhance the capturing, storage, retrieval, transfer and dissemination of knowledge generated as part of ongoing collaborative efforts to improve the new product development and order fulfilment performance of EMEs, as well as:

- Implementation Guidelines supporting the situational design, implementation and ongoing development of collaborative, EME-level improvement, using the Business Model and the Software System.

The business model essentially describes what a collaborative improvement environment might look like, what may be enablers and barriers to achieving such an environment, what possibilities there are to create the enablers and to overcome the barriers. Furthermore, the model proposes and describes tools that are available for the partners to manage and monitor key aspects of the development process. The portal-based software system aims to facilitate collaboration between, and joint learning by, dispersed partners.

The present paper focuses on enablers and barriers to getting collaborative improvement up and running. The research question addressed in the paper is:

What disabling and enabling factors are at play in the process of developing collaborative improvement in an EME environment, how do these factors affect that process and each other?

For reasons of space we decided not to spend much text to presenting and discussing theoretical background and methodological considerations. Rather, we preferred to go straight into the heart of the matter, to present the field research organised according to the most important findings, and to discuss those findings. So, after a few words on method and empirical setting, the main findings are presented, organised according to the factors found to impact the development of collaborative improvement. Next, the findings are discussed. The article is concluded with summary and lines for further research.

\section{METHODOLOGY}

The central methodology in the three-year project is action research (by university teams working closely together with three EMEs) of action learning processes (by the EMEs). In the course of the project, the software is developed and the three EMEs are gradually learning to improve collaboratively and to use the software to support that. The interventions by action researchers are based on the theory assembled in the business model and aimed at improving the collaborative improvement situation all the time.

\section{EMPIRICAL FIELD}

The empirical field consists of three EMEs each comprising one systems integrator and 
three or four suppliers. A System Integrator (SI) is defined as a company that integrates components provided by a number of suppliers. The system integrators are located in Denmark, Italy and The Netherlands and their suppliers in Austria, Denmark, Germany, Italy and The Netherlands. In addition two software vendors (Sweden, Greece) and four universities (Denmark, Italy, Ireland, The Netherlands) are involved. The EMEs are active in the following businesses:

\begin{tabular}{|c|c|c|c|c|c|}
\hline & SI & supplier 1 & supplier 2 & supplier 3 & supplier 4 \\
\hline Denmark & $\begin{array}{c}\text { mobile } \\
\text { hydraulics }\end{array}$ & metal parts & metal parts & $\begin{array}{l}\text { foundry } \\
\text { products }\end{array}$ & \\
\hline Italy & aircraft & $\begin{array}{l}\text { composite } \\
\text { parts }\end{array}$ & metal parts & metal parts & metal parts \\
\hline Netherlands & $\begin{array}{c}\text { electro } \\
\text { hydraulics }\end{array}$ & $\begin{array}{l}\text { plastic } \\
\text { moulding }\end{array}$ & $\begin{array}{l}\text { fine-mechanical } \\
\text { parts }\end{array}$ & cylinder tubes & \\
\hline
\end{tabular}

\section{EMPIRICAL FINDINGS}

This section describes the factors affecting the development of collaborative improvement in the three EMEs. The research confirms the role of some factors already identified in the literature:

- Vision, i.e. sense of direction (DiBella \& Nevis 1998).

- Commitment (Monczka et al. 1998, Mohr \& Spekman 1994, Moore, 1998).

- (Relative) power (Buchanan \& Badham 1999, Cook 1977).

- Trust (McCutcheon \& Stuart, 2000, Sako 1992).

In addition, however, the study also identified a significant role for other endogenous factors, in particular, approach taken, project organisation, competencies of the people directly involved, communication, and political behaviour. Influential factors exogenous to the collaboration include national culture and joint history.

\section{The Danish EME}

In the Danish EME we found some commonality between the system integrator (SI) and suppliers 1 and 2 (dyads 1 and 2) whereas the process was different for the SI and supplier 3 (dyad 3).

Vision. The SI had a clear vision on the development of their supply chain. They intended to roll out its well-developed Total Productive Maintenance (TPM) into the supply chain, and also wished to establish strategic collaboration with the three suppliers. However, all four companies lacked a clear vision and a deep understanding of collaborative improvement and the prerequisites for and consequences of that.

Approach. The approach towards collaborative improvement can be characterised as learning-by-doing initiated by improvement projects. The idea behind this approach was that the companies would complete a number of improvement projects together and from this develop a more mature collaborative improvement environment, by:

B Spreading the new knowledge deeper into, and further up into their organisations.

B Taking the new knowledge to a higher learning level.

B Developing a routine of continuous improvement between the companies. 
B Increasing more fundamental, larger scale, more complex and higher-impact improvements.

The lack of vision influenced the suppliers' approach, as they were not able to foresee the consequences and remained hesitant well into the project. As a consequence, the researchers had to put much more effort into facilitating the collaboration than envisaged. An intervention by the researchers triggered a meeting at which vision was the key item on the agenda. After the meeting, independence and confidence were high enough for the EME to take over, so that the researchers could withdraw on a more research oriented role.

Project organisation (and culture). Initially, progress was heavily dependent on researcher facilitation, both at monthly workshops and between those meetings. The workshop agendas were set by the researchers. At the workshops the companies identified a number of relatively small-scale improvement projects: short, aimed at quick success, and involving only a few people. Small groups, consisting of a researcher, a purchaser from the SI and the responsible person from the suppliers (primarily managing directors), would identify improvement projects, work out a project plan and present this in plenum. This way of running workshops came very natural to the participants because this is the typical culture of workshops in Denmark. In between workshops the researchers interacted with all companies on a weekly basis to facilitate the improvement projects and make the collaboration more mature. Communication between the companies naturally evolved around these improvement projects, but was highly influenced by (lack of) improvement competence, and also by political behaviour.

Competence and commitment. The EME members lacked improvement and change competence. None of the suppliers had previous experience with continuous improvement, while the SI's ample experience with TPM was limited to intra-organisational settings. Most importantly, though, none of the persons involved (the SI's purchasers and the suppliers' MDs) were experienced change agents or (improvement) project managers. This was detrimental to both the small improvement projects as well as the learning from these activities needed to create a more mature collaborative improvement environment.

Communication. The way dyads 1 and 2 communicated can be characterised as "closed books" with hidden agendas and strongly influenced by political behaviour. The suppliers in these dyads were uncertain of the SI's motives to invite them into CO-IMPROVE. They showed their uncertainty by being dishonest with the SI and not too eager to identify improvement projects. The hidden agendas came to the surface when researchers visited the companies without any of the other partner being present. The communication in dyad 3 was much more open, honest and all participants had a win-win attitude. The reason for this are described further on in this section.

Power. The SI has throughout history dominated suppliers by focusing on cost reductions and this equipped the SI with a certain power of the suppliers. Supplier 2 and 3 depended on the SI to the extent that they delivered a large portion their total turnover to that customer. Supplier 1 hardly depends on the SI for its sales.

Trust (and history). Initially, the trust between SI and supplier 1 and 2 was quite low. This was due to history in which the SI had first removed large portions of turnover from these suppliers and then gradually given it back to them. Mistrust further increased because of the SI was quite ambiguous about its motives. Again, the suppliers were not sure why the SI invited them to participate in CO-IMPROVE. Supplier 2 even thought the reason was to 
get insight in the supplier's core foundry competencies, which they suspected the SI would use to find alternative suppliers. A history of good relationship has on the other hand increased the trust relation between SI and supplier 3 to a high level. The relationship is based on personal friendship between the owner of the company, and the purchaser and plant manager at the SI. The two companies have always collaborated in a very open way. The dependence of the supplier on the SI for its turnover has steadily increased, to such a high level, though, that it is becoming unhealthy for that company.

Political behaviour: In dyads 1 and 2 lack of trust from the suppliers, power attitude from the SI and lack of vision from all companies created political behaviour. The suppliers felt insecure of the situation and project, acted reluctantly, but wanted to appear interested. The SI more or less forced them to join and stay in CO-IMPROVE. In dyad 3, the relative power from the SI and lack of vision between all partners did not create political behaviour, due to the high level of trust between the partners.

Conclusion

In effect, dyads 1 and 2 experienced major dips in the process towards collaborative improvement and the relationship has only slightly improved. Dyad 3 experienced a steady process and the relationship has improved even further. The companies have in total initiated 13 improvement projects, 12 are ongoing and 1 is suspended.

\section{The Dutch EME}

Vision. Continuous improvement and continuous cost reduction are integrated and explicit in the SI's policy and practices. The aim is to establish close co-operation and long-term agreements with a limited number of suppliers. As such, the SI looks for highly involved and dedicated partners that fully support the company in assembling and delivering to customers systems of top quality to agreed competitive prices at the promised delivery date. However, the suppliers' initial expectation was that the project involved just another way of imposing cost reductions and other improvements. This had a major impact on how they acted. In fact, they actually waited for initiatives from the SI, and did not show any sense of urgency.

Approach (and culture). The Dutch EME took a 'laissez-faire' approach towards collaborative improvement. The idea was that the companies involved would jointly initiate improvement projects in the same way as in the Danish EME. After three months however, hardly any improvement projects had started as none of the EME participants would take the initiative and because all lacked a sense of urgency. The SI and researchers decided to change the approach towards a more active (directive) role of the SI, and approach that fits much better also to the Dutch culture of doing business.

Project organisation. The initial idea was to start with dyad-level improvement projects, which were identified through interviews with representatives of the companies and the results of operational integration and collaborative improvement maturity assessments. After a period of no results, the frequency of workshops increased from half a day every two months to a full day every month in order to increase the efficiency and effectiveness of the meetings. The SI has taken on a more active role with regard to selecting and monitoring the progress of the improvement projects. The companies themselves are responsible for the content, planning and execution of the projects. The researchers are facilitating the companies by providing guidelines for improvement and helping companies 
extract lessons and improve their collaborative improvement activities.

Competence and commitment (and history). The suppliers involved in the CO-IMPROVE project had a joint history of working together with and for the SI on improvement projects. So, they did possess the improvement knowledge and skills required. However, it appeared difficult for them to diffuse the knowledge and skills, generated within and through different projects, into their respective organisations, and to share learning moments and experiences.

Communication. A common ground for improvement was provided by the systems integrator's system of supplier assessment (on quality, cost and delivery) and when trust started to build up slowly within the EME, more open ways of communication and information sharing became evident.

Power. For a company in the automotive industry today the main challenge is to constantly monitor the cost-structure in order to remain profitable. The SI has to and does forward cost reductions to the suppliers, and they can do so, as, at least for two of the three suppliers, the SI is one of their biggest customers. However, the SI has explicitly chosen, at least initially so, not to be too directive and to prescribe improvement projects, considering that such a position would not facilitate voluntary participation of the suppliers in the development of collaborative improvement. When this approach appeared to fail, the SI imposed a, what appeared to be much more successful, 'directed laissez-faire' approach.

Trust (and history). Trust between the partners depended very much on personal relationships between employees from the suppliers and the SI. For all of the three suppliers these personal relationships can be described as very good with a high level of personal commitment and open ways of communication. Further into the project, the level of trust was positively affected by the communication in the workshops, willingness to participate in discussions, to provide information, to reflect on activities and to draw up lessons learned.

Political behaviour (and history). There has not been any proof of political behaviour, which is probably due to the high trust relationship and the fact that SI had no intention of using its possible power.

Conclusion

After a slow start the EME really got on track. The companies energetically started to work collaboratively and initiated five improvement projects of which at date two have been finished successfully. The success mainly is reflected in the lessons that the companies have extracted after finalising the improvement projects. The Dutch EME now seems to have found a successful approach towards collaborative improvement, which can be characterised as a 'directed laissez-faire' approach.

\section{The Italian EME}

Some of the factors did not show as clearly as in the other two EMEs and the initial approach towards collaborative improvement was different as well.

Vision (and history). The industry is characterised by long-term partnerships. In spite of that, there was a lack of joint vision and deeper understanding of collaborative improvement. 
Approach. In order to test the influence of different contingent factors, the SI selected four suppliers, which differed in terms of size (two small, one medium and one large company), product (two simple and two complex), and location (two very close, one in a nearby region and the fourth in a different country). Focussing on the concept of collaboration, the project started with a retrospective analysis of the dyads involved in the EME. The researchers collected histories of past attempts of collaborative improvements, and discussed them separately with the five partners in order to find out barriers to collaboration while avoiding biases related to different bargaining power positions. The first three workshops were dedicated to introducing concepts of collaborative improvement and supply chain collaboration, through assessment exercises, so as to create an environment suitable to collaborative improvement initiatives.

Project organisation. The action research process was structured with a series of one-day monthly workshop with a well-defined agenda and assignments in between. The SI selected a core team formed by representatives from different departments (Quality, Continuous Improvement, Engineering, Manufacturing, Purchasing, ICT) led by a senior manager from the Purchasing department. Suppliers participated with a sales manager and one or two representatives from either manufacturing or engineering. At the workshops the academics took the lead, acting as methodologists and facilitators, while the purchasing manager kept himself hidden in the background. Progress was slow and no results were achieved, so a change of structure was made. Starting from the third meeting, subgroups, each formed by a supplier and representatives from the SI, were encouraged to generate and select ideas for pilot collaborative improvement initiatives and to plan related activities. Presentation and discussion of results boosted commitment as both the suppliers as well as the SI representatives were proud to show measurable results. Even though the researchers tried to shift attention from results from specific initiatives to results in terms of learning on how to collaborate at EME level, the main attention was still on the former.

Competence and commitment. The EME had a high level of managerial and technical skills. The SI involved a continuous improvement manager, and two of the suppliers had high levels of management and technical competence. Dyads 1 and 2 were the most successful ones. One of the two sons of the owner of supplier 1 participated in the project with high energy and a positive management style. The key representative from the SI was the person responsible for Kaizen activities, who also gave a strong contribution in terms of method and ability to involve experts from the company. The level of managerial and technical competence of supplier 2 was high and both companies in the dyad perceived learning benefits in working together. Due to organisational problems at supplier 4, the project lacked attention, which negatively affected the level of improvement. Furthermore, a change in that supplier's representatives in the group inhibited building mutual trust.

Communication. Initially, the workshops were characterised by a certain degree of difficulty in communication and low the level of openness but this improved significantly after the change of approach indicated above. Line representatives of the suppliers and the SI played the main roles with a decreasing need for facilitation by the researchers. Although distance and language differences between the SI and supplier 2 made communication more difficult, the level of collaboration was good, particularly after results were reached.

Power. For companies in the aircraft business, sustained top performance is a matter of life 
or death, and they have to demand the same from their suppliers. However, the SI deliberately chose to go for learning from the project, rather than imposing new practices and improvement levels on its suppliers.

Trust (and history). Trust in dyads 1 and 2 was good, but more problematic in dyads 3 and 4. The level of trust had a clear effect on the speed at which collaborative improvement came off the ground and results were achieved. In dyad 1, mutual trust and, through that, communication was immediately good and the project generated improvements not only between the companies, but even more within the SI. Supplier 3 is a small company and very dependent on the SI. Initially, the owner participated; later the sales manager joined the project. Negotiation and previous difficulties made the relationship and communication quite defensive. However, when the first improvement results arrived, trust and also commitment began to increase.

Political behaviour. Except in dyad 1, the level of commitment in the first stage was low and people appeared to be defensive and sceptical about the real value of the project. At that stage the role of the purchasing manager was fundamental in preparing the core team and motivating suppliers to participation. In the period between meetings researchers had to play the key role in planning activities, organising meetings and taking part of the political issues by pressing people to work on assignments and stick to due dates.

\section{Conclusion}

The EME initiated the project with assessment and goal alignment, but did not see any significant results. The approach changed to evolve around improvement projects facilitated by researchers and highly competent continuous improvement managers and has started one improvement project per dyadic relationship. This quickly led to good results in dyads 1 and 2, while the other two picked up more slowly.

\section{DISCUSSION}

Figure 1 shows the factors affecting the development of collaborative improvement, and illustrates how they influence each other.

Although all these factors seem to play a significant role, their influence may be entirely different. In the Dutch EME power and trust were at such a high level that these factors acted as enablers. In contrast, the Danish case describes a heavy load of political behaviour because of lack of trust and vision combined with a powerful position of the SI. The project organisation followed the approach and national culture, and seemed to have a strong enabling effect in the Danish EME. That is, the approach actually generated tangible improvements, which took the focus somewhat away from disabling factors such as political behaviour and lack of competencies. In the Dutch EME, a similar approach was chosen. However, here the focus on small-scale improvement projects did not create the same results. The reason can be found in the level of facilitation by researchers, which visited and worked with the companies on a weekly basis, while contact with the Dutch EME partners was much less frequent. Also, in Denmark monthly workshops were held while the Dutch ones took place every two months. This created different levels of awareness, commitment and focus. In Italy, the decision to introduce collaborative improvement through goal alignment and a range of assessments did not create immediate results. This could indicate that results in the initial phase are generated from high levels of facilitation and frequent workshops to create awareness and momentum. 


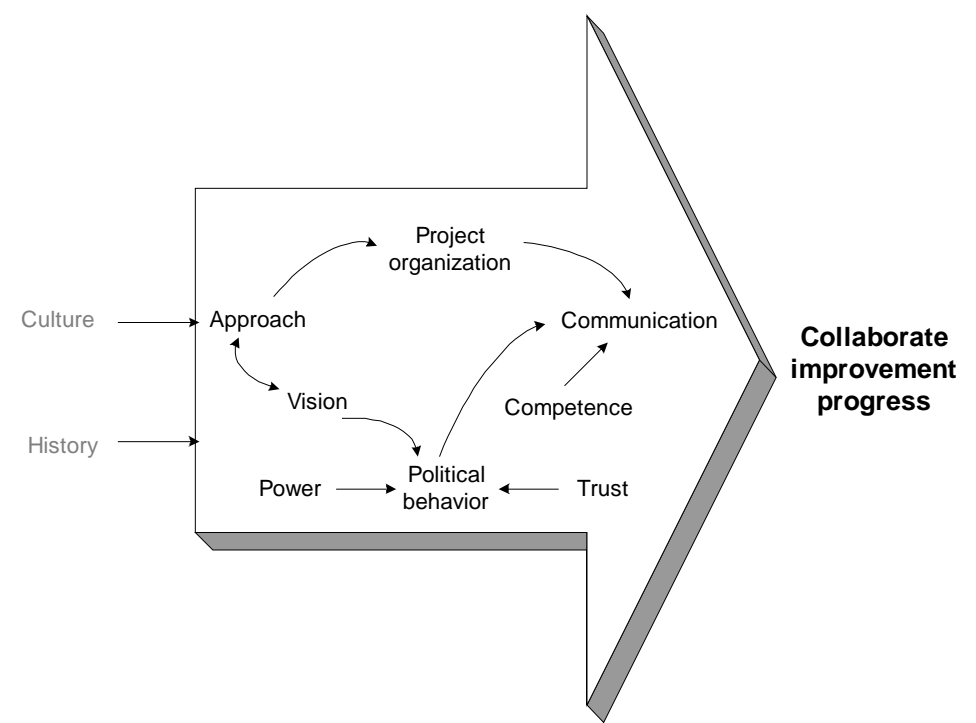

Figure 1 Factors at play in the development of collaborative improvement.

\section{CONCLUSION}

The objective of the paper was to identify factors enabling and disabling the process of getting collaborative improvement in an EME environment up and running, and to investigate how do these factors influence each other. Three system integrators with three to four suppliers each provided the empirical setting. Through the three cases a number of endogenous and exogenous factors are identified, see Figure 1.

Some of these factors are not really new. In particular, vision, i.e. sense of direction (DiBella and Nevis 1998), commitment (Monczka et al. 1998, Mohr and Spekman 1994, Moore, 1998), (relative) power (Buchanan and Badham 1999, Cook 1977) and trust (McCutcheon and Stuart, 2000, Sako 1992) have been described in previous publications. Other factors are new and include approach taken, project organisation, competencies of the people directly involved, communication, and political behaviour. What is particularly important is:

- The interaction of (some of) the factors. It is not so much individual factors, but the configuration of factors that determines the development of collaborative improvement.

- Within any configuration, the same factors can be beneficial and detrimental to collaborative improvement, dependent on the setting in which they play their respective roles.

The same factors showed to play a role in all EMEs. However, some of them can be a disabler in one EME and an enabler in another EME. Furthermore, the negative influence of disablers can be overcome by the positive influence of others. The Danish case has initiated 13 improvement projects in spite of disabling factors such as strong political behaviour, a dominant position of the system integrator and lack of shared vision. The enabling factors were found to be the approach, i.e. learning-by-doing, high frequency of interactions and high level of facilitation by researchers. The Dutch EME has initiated five 
improvement projects enabled by factors such as high trust level and low power distance between partners. Disabling factors were a laissez-fair approach that did not lead to results, and low level of workshop frequency. The Italian case has initiated four improvement projects and did not experience many results in the first phase of the project due to disablers such as communication and political behaviour. The enabling factors in the Italian case were highly competent company members. A change of approach (presentation and discussion of results) to the project appeared to boost progress.

Further research is needed to find out which of the three (four) approaches (bottom-up learning-by-doing, '(directed) laissez-faire', top-down creation of an environment suiting collaborative improvement) is the most successful on the long run. Furthermore, there is no doubt that the research identified the most important factors affecting the initial (relative) success of these approaches, but further research is needed to be able to infer guidelines for other EMEs intending to engage in the adventure called collaborative improvement.

\section{REFERENCES}

AMR Research (1999), Integrated supply chain planning is not for everyone yet, September.

Boer, H., A. Berger, R. Chapman and F. Gertsen (2000), CI changes. From suggestion box to organizational learning. Continuous improvement in Europe and Australia. Ashgate, Aldershot.

Buchanan, D., and R. Badham (1999), Power, politics and organizational change. Winning the turf game, Sage, London.

Busby, Fan (1993), "The extended manufacturing enterprise: its nature and its needs", International Journal of Technology Management, Vol. 8, No. 3-4-5.

Cook (1977), "Exchange and power in networks of inter-organizational relationships", Sociological Quarterly, Vol. 18.

Coughlan, P. and D. Coghlan (2002), "Action research for operations management", International Journal of Operations \& Production Management, Vol. 22, No. 22, pp. 220-240.

DiBella, A.J. and E.C. Nevis (1998). How organizations learn: An integrated strategy for building learning capability. San Francisco: Jossey-Bass.

Frohlich, M.T. and Westbrook, R. (2001), "Arcs of integration: an international study of supply chain strategies", Journal of Operations Management Vol. 19, pp. 185-200.

Ghoshal, S. and C. Bartlett (1995), "Changing the role of top management: Beyond structure to processes", Harvard Business Review, January-February, pp. 75-87

Hayes, R.H and S.C. Wheelwright (1984), Restoring our competitive edge competing through manufacturing, John Wiley \& Sons, New York.

Kumar, N. (1996), "The power of trust in manufacturer-retailer relationships", Harvard Business Review, Vol.74, No.6, pp. 92-106.

McCutcheon, D. and F.I. Stuart (2000), "Issues in the choice of supplier alliance partners", Journal of Operations Management, No.18, pp.279-301

Middel, R., R. Cagliano, F. Caniato, R. Kaltoft and J.S. Nielsen (2003), "Challenging EMEs to learn through collaborative improvement projects", EurOMA conference, Como (forthcoming).

Michigan State University, Data collection from the Annual Executive Purchasing and Supply Chain Management seminar, East Lansing Michigan, 1990-2000.

Sako, M. (1992), Prices, quality and trust: inter-firm relations in Britain and Japan, Cambridge University Press, Cambridge.

Stock G. N., N.P. Greis and J.D. Kasarda (2000), "Enterprise logistics and supply chain structure: the role of fit", Journal of Operations Management, No.18, pp. 531-547. 